\title{
Goodness-of-Fit Model-Dependent Approach for Release Kinetics of Levofloxacin Hemihydrates Floating Tablet
}

\author{
V.T.Thakkar', ${ }^{1,3}$, P. A. Shah'1, T. G. Soni ${ }^{1}$, M. Y. Parmar ${ }^{1}$, M. C. Gohel ${ }^{2}$, and \\ T. R. Gandhi' \\ 'Department of Pharmaceutics, Anand Pharmacy College, Anand- 388001 Gujarat, INDIA \\ ${ }^{2}$ Department of Pharmaceutics, L.M. College of Pharmacy Ahmedabad-380009, Gujarat, INDIA
}

e-mail:vtthakkar@rediffmail.com

\begin{abstract}
The objective of this work was to develop floating levofloxacin tablets and to understand the kinetics of drug release by applying mathematical and model-dependent approaches. Nine formulations of floating tablets were prepared by the direct compression method using Gelucire 43/01 (hydrophobic) and hydroxypropyl methylcellulose (hydrophilic) as matrix-forming excipients. The in vitro drug release was studied in $\mathrm{pH} 1.2 \mathrm{HCl}$ using USP dissolution Apparatus 2 at 50 rpm. Zero-order, first-order, Higuchi, Hixson-Crowell, and Korsmeyer et al. models were used to estimate the kinetics of drug release. The criteria for selecting the most appropriate model were based on the goodness-of-fit test and lowest sum of squares residual. Drug release from the optimal batch was explained by the Higuchi model. A simple mathematical approach was applied to determine the deviation in area under the curve (AUC) between predicated and observed dissolution data. The difference in percent deviation of AUC at each point was lowest for the optimum batch. Drug release was a function of the ratio of hydrophobic to hydrophilic matrixing agent.
\end{abstract}

\section{INTRODUCTION}

$\mathrm{n}$ a relatively short time, Helicobacter pylori (H. pylori) has become recognized as a major gastric pathogen with worldwide distribution (1). H. pylori, a prevalent human-specific pathogen, is a causative agent in chronic active gastritis, duodenal ulcers, gastric adenocarcinoma, and one of the most common forms of cancer in humans $(2,3)$. It should be susceptible to many antibiotics in vitro but has been proved difficult to eradicate in vivo (4). Extended resident time of the antimicrobial agent is desirable for more effective eradication of $H$. pylori $(1,5)$. To extend the residence period, several approaches have been developed such as floating drug delivery systems, swelling and expanding systems, polymeric bioadhesive systems, modified-shape systems, high-density systems, and other delayed gastric emptying devices (6-8).

Levofloxacin hemihydrate (Levo), a synthetic fluorinated quinolone derivative, is effective for bacterial infection treatment, especially for H. pylori (9-12). The failure of therapy can be avoided by providing the effective concentration of antibiotics at the site of action (13). In this study, an attempt was made to formulate Levo floating tablets with the use of low density polymer hydroxypropyl methylcellulose (HPMC K4M) and the release-retarding hydrophobic polymer Gelucire 43/01 $(14,15)$. Gelucire, a mixtures of mono-, di-, and triglycerides with polyethylene glycol (PEG) esters of fatty acids, was used in combination with the hydrophilic polymer

${ }^{3}$ Corresponding author.
(HPMC K4M) to minimize the hydration rate of the floating tablet and variability in the release profiles of the highly water soluble drug (Levo). The floating matrix tablet swells upon ingestion and a gel layer forms on the surface of the tablet. This gel layer slows the entry of dissolution fluid and release of drug. It is a proven fact that drug release from hydrophilic matrices shows a typical time-dependent profile (i.e., decreased drug release with time because of increased diffusion path length) (16). Higuchi tried to relate drug release rate to physical constants based on simple laws of diffusion, which is shown in following equations.

$$
Q=\left[D\left(2 C-C_{s}\right) C_{s} t\right]^{1 / 2}
$$

where $Q$ is the amount of the drug released in time $t$ per unit area, $C$ is the initial drug concentration, $C_{s}$ is the drug solubility in the matrix media, and $D$ is the diffusivity of the drug molecules in the matrix substance.

The simplified form of eq 1 is

$$
Q=K_{H} t^{1 / 2}
$$

where $K$ is the Higuchi dissolution constant.

Equation 2 was used to determine the Higuchi release rate constants for different formulations. Regression analysis was adopted to compute the constants. Gohel et al. proposed a mathematical approach for the determination of deviation in Higuchi release profile (17). The rationale behind this study was to select an optimized batch by evaluating the deviation of dissolution data using an AUC-based mathematical approach as well as goodness of fit to the kinetic models. 


\section{EXPERIMENTAL \\ Materials}

Levofloxacin hemihydrate was received as a gift from Marck Biosciences Ltd. (Kheda, India). Hydroxypropyl methylcellulose (HPMC K4M) was a gift from Colorcon Asia Pvt. Ltd. (Goa, India). Gelucire 43/01 (waxy solid, melting point $43^{\circ} \mathrm{C}, \mathrm{HLB}=01$ ) was a gift from Gattefosse (St. Priest, Cedex, France). Sodium bicarbonate, talc, and magnesium stearate (analytical grade ) were purchased from S. D. Fine Chemicals Ltd. (Mumbai, India). All other ingredients used in the study were of analytical grade.

\section{Methods}

Preparation of Levofloxacin Hemihydrate Floating Tablet Levo, HPMC K4M, and Gelucire 43/01 were passed through a No. 80 sieve separately. Drug was mixed with the polymers and other ingredients as listed in Table 1. All batches contained 50\% (w/w) Levo, 2\% (w/w) talc, and 1\% $(\mathrm{w} / \mathrm{w})$ magnesium stearate; the average weight of each tablet was $500 \mathrm{mg}$. The powder blend was then lubricated with magnesium stearate and talc, and this lubricated blend was compressed into tablets using 16-mm flat-face round tooling on a Minirotary tablet press (Cadmach, Ahmedabad, India). The compression force was adjusted to obtain tablets with hardness in the range of $5-6 \mathrm{~kg} / \mathrm{cm}^{2}$. The matrix tablets, batches F1 to F9, were prepared by direct compression according to the formulas depicted in Table 1.

\section{Calibration Curve Preparation}

A stock solution of Levo (100 mg/mL) was prepared in $0.1 \mathrm{~N} \mathrm{HCl}$. It was further diluted to obtain the known standard solutions in the range of $1-10 \mu \mathrm{g} / \mathrm{mL}$. Hydrochloric acid $(0.1 \mathrm{~N} \mathrm{HCl}, \mathrm{pH} 1.2)$ was prepared by adding $8.5 \mathrm{~mL}$ concentrated acid to $991.5 \mathrm{~mL}$ of double-distilled water with cooling. The absorbance was measured spectrophotometrically (Shimadzu UV/Vis spectrophotometer 2100, Tokyo, Japan) at $293 \mathrm{~nm}$ with the mean data $(n=6)$ used for the calibration curve. The concentrations of dissolved drug in the formulations were calculated from the regression equation obtained from the calibration curve.

\section{Dissolution Study of Prepared Formulations}

Dissolution studies of prepared floating tablets were conducted using USP Apparatus 2 (paddle). The dissolution medium was $900 \mathrm{~mL}$ of $0.1 \mathrm{~N} \mathrm{HCl}$ at $37 \pm 0.5^{\circ} \mathrm{C}$ at $50 \mathrm{rpm}$. The dissolution study was performed on batches F1 to F9. Sample aliquots $(5 \mathrm{~mL})$ were withdrawn at $1,2,3,4,5,6,7,8,9$, and 10 hours and replaced with equal volumes of fresh medium. Drug content was determined spectrophotometrically at $293 \mathrm{~nm}$. The mean data $(n=3)$ were used. Percentage cumulative drug release $(\% \mathrm{CDR})$ was plotted against time.

\section{Model-Dependent Approaches}

In vitro drug release data were fitted to kinetic models such as zero-order (18), first-order (19), Higuchi equation (16), Korsemeyer-Peppas equation (20), and HixsonCrowell equation (21). The regression analysis was performed.
$Q_{t}$ versus $t$

$\log Q_{t}$ versus $t$

$Q_{t}$ versus square root of $t$ $\log \% Q_{t}$ versus log $\% t$

$Q_{t}$ versus cube root of $t$

\author{
(zero order) \\ (first order) \\ (Higuchi) \\ (Korsmeymer-Peppas) \\ (Hixson-Crowell)
}

where $Q_{t}$ is the amount of levofloxacin released at time $t$. The criteria for selecting the most appropriate model were sum of square of residuals (SSR) and Fischer's ratio.

\section{Mathematical Approach}

A novel approach of deviation from Highchi model proposed by Gohel et al. (17) was used to evaluate deviation between predicted and observed dissolution profile of floating formulations. The predicted percentage CDR at each time point was back-calculated using the regression equation of Higuchi model. Predicted percentage of drug released versus square root of time is considered as a reference line. AUC at $0 \%$ deviation for the predicted and observed data was calculated by using eq 3 . The entire dissolution profile was compared by taking the absolute difference (residual) between the predicted and observed calculated AUC data.

Table 1. Tablet Formulations for Preliminary Trials

\begin{tabular}{|c|c|c|c|c|c|c|c|c|c|}
\hline \multirow[b]{2}{*}{ Ingredient } & \multicolumn{9}{|c|}{ Batch } \\
\hline & $\mathbf{F 1}$ & $\mathbf{F 2}$ & F3 & F4 & F5 & F6 & F7 & F8 & F9 \\
\hline Levofloxacin hemihydrate (mg ) & 250 & 250 & 250 & 250 & 250 & 250 & 250 & 250 & 250 \\
\hline Gelucire 43/01 (\% ) & - & 5 & 10 & 15 & 20 & 25 & 15 & 18 & 40 \\
\hline Methocel HPMC K4M (\%) & 40 & 35 & 30 & 25 & 20 & 15 & 35 & 22 & - \\
\hline Sodium bicarbonate (\%) & 10 & 10 & 10 & 10 & 10 & 10 & 10 & 10 & 10 \\
\hline Floating lag time (sec) & 425 & 389 & 352 & 258 & 385 & 464 & 375 & 312 & 465 \\
\hline
\end{tabular}

* HPMC: hydroxypropyl methylcellulose. 


$$
\begin{aligned}
& A \cup C_{t h r, 0 \% \text { deviation }}=\left(\frac{k_{H} \sqrt{t}+k_{H} \sqrt{t-n}}{2}\right) \times(\sqrt{t}-\sqrt{t-n}) \\
& A U C_{t h r, 0 \% \text { deviation }}=\frac{k_{H}}{2} n
\end{aligned}
$$

where $k_{H} t$, and $n$ are the Higuchi rate constant, time, and difference between two successive sampling time points, respectively.

Residual values between predicted and observed data were used to calculate the sum of squares of residuals as well as mean sum of square values for optimized batch among all the models.

\section{RESULTS AND DISCUSSION}

Direct compression was adopted considering its advantages such as simple technology and saving of time. The tablets of levofloxacin hemihydrate were prepared by direct compression using HPMC K4M, Gelucire 43/01, and sodium bicarbonate. Magnesium stearate and talc were used as lubricant and glidant, respectively. Nine batches (F1-F9) were prepared as described in Table 1. The average table weight was $515 \mathrm{mg}$, and the weight variation for every batch was less than $\pm 4 \%$. The crushing strength of the tablet was between 4 and $6 \mathrm{kgf}$ and was maintained for all batches to minimize the effect of crushing strength on drug release. Drug content uniformity in all formulations was calculated and was found satisfactory. Friability of all the formulations $(<1 \%)$ showed satisfactory resistance to mechanical shock and abrasion. Sodium bicarbonate was used as gas-generating $\left(\mathrm{CO}_{2}\right)$ agent. The generated gas was trapped within the gel (formed by hydration of HPMC K4M) which facilitated floating due to density reduction. The tablets exhibited a short floating lag time due to the presence of sodium bicarbonate. The floating lag time ranged from 258 to 464 $\mathrm{sec}$, indicating the importance of formulation variables. The difference in floating lag time could be attributed to the difference in fluid uptake and subsequent swelling. Gelucire 43/01 was selected as a release-retarding agent. HPMC K4M was selected as a matrixing agent; it is nontoxic and has excellent gelling and sustained-release properties. The tablets containing a high percentage of HPMC K4M remained floating for a longer duration than formulations containing a higher percentage of Gelucire 43/01. In vitro dissolution of batches F1 to F9 was performed in dissolution media with a $\mathrm{pH}$ 1.2. The drug release profiles of batches F1 to F9 are shown in Fig. 1. Gelucire 43/01 was selected as a release-retarding agent, which minimized the variation in the release profile of highly water soluble drugs (15). HPMC K4M was selected as a matrixing agent because of its excellent gelling and sustained-release properties. Sodium bicarbonate generated $\mathrm{CO}_{2}$ gas with hydrochloric acid present in the dissolution medium. The generated gas might be trapped within the gel (formed by hydration of HPMC K4M), which may decrease the density of the tablet below 1 .

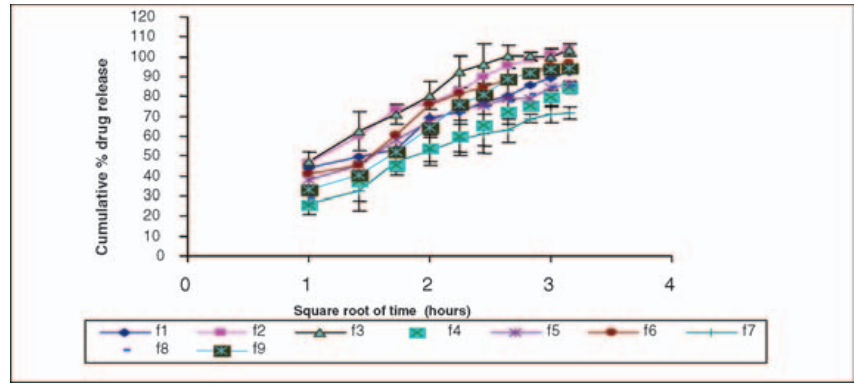

Figure 1. Cumulative $\%$ drug release vs. square root of time for batches F1-F9.

In vitro dissolution testing of batches F1-F9 was performed using $\mathrm{pH} 1.2$ dissolution media. The drug release profiles are shown in Figure 1. To investigate the drug-release kinetics, data were fitted to various kinetic models such as zero-order, first-order, Higuchi equation, Korsemeyer-Pappas equation, and Hixson-Crowell equation. The regression analysis was done for all nine batches. The Higuchi square root model showed higher correlation coefficient values for batch F4 $\left(r^{2}=0.9984\right)$ than for other batches. Residual values were used to compare best fit of the observed data to the predicted. The mean sum of square residual values between predicted and observed data of batches F1-F9 were calculated by ANOVA analysis. The mean sum of squares for batch F4 (MSS =185.42) was lowest compared with the other batches. It may be concluded that the minimum variance was found between the predicted and observed dissolution data for batch F4. Table 2 shows the correlation of batch F4 to various kinetic models. The highest value of $r^{2}(0.9982)$ and lowest sum of square residuals ( $S S R=4.96$ ) was found for batch F4 with the Higuchi model. Table 3 shows the absolute difference between observed and calculated AUC based by simple mathematical approach for batch F4. Drug is released by a diffusion mechanism. The results can be used for setting IVIVC.

\section{Table 2. Results of Model Fitting for Levofloxacin Hemihydrates (Batch F4)}

\begin{tabular}{lcccc}
\hline Model & \multicolumn{1}{c}{$\boldsymbol{r}^{\mathbf{2}}$} & SSR & Slope & Intercept \\
\hline Zero order & 0.9756 & 88.34 & 59.719 & 20.67 \\
\hline First order & 0.9724 & 176.2 & 6.2679 & 25.359 \\
\hline Higuchi & 0.9987 & 4.044 & 27.113 & -1.091 \\
\hline Hixson-Crowell & 0.9892 & 33.99 & 53.257 & -29.169 \\
\hline Peppas & 0.971 & 104.2 & 0.4897 & 1.39 \\
\hline
\end{tabular}

$r^{2}$ : square of correlation coefficient

SSR: sum of square residuals 
Table 3. Percentage Deviations for the Test Products from the Ideal 10-h Higuchi Release Profile of Batch F4

\begin{tabular}{|c|c|c|c|c|c|c|}
\hline \multirow{2}{*}{ Time } & \multirow{2}{*}{$\frac{\text { Square root of time }}{\text { Hour }}$} & \multicolumn{2}{|c|}{$\begin{array}{l}\text { Predicted Higuchi Release } \\
\text { Profile }\end{array}$} & \multicolumn{2}{|c|}{$\begin{array}{l}\text { Observed release profile of } \\
\text { levofloxacin tablet }\end{array}$} & \multirow{2}{*}{$\begin{array}{c}\begin{array}{c}\text { Absolute difference } \\
\text { in AUC }\end{array} \\
A U C\end{array}$} \\
\hline & & CPR & $A U C$ & CPR & AUC & \\
\hline 1 & 1.0 & 26.022 & 13.11 & 25.68 & 12.84 & 0.27 \\
\hline 2 & 1.41 & 37.13 & 13.16 & 37.25 & 13.2 & 0.04 \\
\hline 3 & 1.73 & 45.81 & 13.23 & 45.23 & 13.07 & 0.16 \\
\hline 4 & 2.0 & 53.13 & 13.28 & 53.84 & 13.46 & 0.18 \\
\hline 5 & 2.24 & 59.64 & 13.31 & 59.74 & 13.33 & 0.02 \\
\hline 6 & 2.45 & 65.33 & 13.33 & 65.23 & 13.31 & 0.02 \\
\hline 7 & 2.65 & 70.75 & 13.34 & 72.36 & 13.65 & 0.31 \\
\hline 8 & 2.83 & 75.63 & 13.36 & 75.23 & 13.29 & 0.07 \\
\hline 9 & 3.0 & 79.91 & 13.31 & 79.45 & 13.24 & 0.07 \\
\hline 10 & 3.16 & 84.58 & 13.38 & 84.32 & 13.34 & 0.04 \\
\hline
\end{tabular}

CPR: cumulative percentage drug released.

AUC: area under the curve.

\section{CONCLUSION}

Release kinetics is an integral part of formulation development because if the kinetics of drug release is known, one can also advance for the establishment of in vivo in vitro (IVIVC) correlation. Gohel et al. proposed a simple mathematical approach one of scientific methods to optimize and evaluate the error in terms of deviation in $A \cup C$ to the release profiles of formulated products during the formulation development stage. Academicians and researchers may adopt this method because of its simplicity and their inter-relationships may minimize the number of trials in final optimization, thereby improving the formulation development process.

\section{REFERENCES}

1. Warren, J. R.; Marshall, B. Unified curved bacilli on gastric epithelium in active chronic gastritis. Lancet 1983, 321, 1273-1275.

2. Megraud, F.; Lamouliatte, H. Helicobacter pylori and duodenal ulcers. Digest. Dis. Sci. 1992, 37, 769-772.

3. Forman, D.; Webb, P.; Parsonnet, J. H. pylori and gastric cancer. Lancet 1994, 343, 243-244.

4. Evans, D. G.; Evans, D. J.; Graham, D.Y. Adherence and internalization of Helicobacter pylori by HEp-2 cells. Gastroenterology 1992, 102 (5), 1557-1567.

5. Shah, S.; Qaqish, R.; Patel, V.; Amiji, M. Evaluation of the factors influencing stomach-specific delivery of antibacterial agents for Helicobacter pylori infection. J. Pharm. Pharmacol. 1999, 51, 667-672.

6. Moes, A. J. Gastroretentive dosage forms. Crit. Rev. Ther. Drug 1993, 10, 143-195.
7. Talukder, R.; Fassihi, R. Gastroretentive delivery systems: a mini review. Drug Dev. Ind. Pharm. 2004, 30, 1019-1028.

8. Whitehead, L.; Fell, J. T.; Collett, J. H.; Sharma, H. L.; Smith, A. M. Floating dosage forms: an in vivo study demonstrating prolonged gastric retention. J. Controlled Release 1998, 55, 3-12.

9. Dicaro, S.; Zocco, A. M.; Cremonini, F.; Candelli, M.; Nista, E. C.; Bartolozzi, F.; Armuzzi, A.; Cammarota, G.; Santarelli, L.; Gasbarrini, A. Levofloxacin based regimens for the eradication of Helicobacter pylori. Eur. J. Gastroenterol. Hepat. 2002, 14 (12), 1309-1312.

10. O'Morain, C. Treatment of Helicobacter pylori Infection. Eur. Gastroenerol. Rev. 2005, 1, 56-59.

11. Watanabe, Y.; Aoyama, N.; Shirasaka, D.; Maekawa, S.; Kuroda, K.; Miki, I.; Kachi, M.; Fukuda, M.; Wambura, C.; Tamura, T.; Kasuga, M. Levofloxacin based triple therapy as a second-line treatment after failure of Helicobacter pylori eradication with standard triple therapy. Digest. Liver Dis. 2003, 35, 711-715.

12. Nista, E. C.; Candelli, M.; Zocco, M. A.; Cremonini, F.; Ojetti, V.; Finizio, R.; Spada, C.; Cammarota, G.; Gasbarrini, G.; Gasbarrini, A. Levofloxacin-Based Triple Therapy in First-Line Treatment for Helicobacter pylori Eradication. Am. J. Gastroenterol. 2006, 101 (9), 1985-1990.

13. Majithiya, R. J.; Murthy, R. S. R. Chitosan-Based Mucoadhesive Microspheres of Clarithromycin as a Delivery System for Antibiotic to Stomach. Curr. Drug Del. 2005, 2 (3), 235-242. 
14. Sutananta, W.; Craig, D. Q. M.; Newton, J. M. An evaluation of the mechanisms of drug release from glyceride bases. J. Pharm. Pharmacol. 1995, 47, 182-187.

15. Shimpi, S.; Chauhan, B.; Mahadik, K. R.; Paradkar, A. Preparation and evaluation of diltiazem hydrochloride-Gelucire 43/01 floating granules prepared by melt granulation. AAPS PharmSciTech 2004, 5, E4.

16. Higuchi, T. Mechanism of sustained action medication: theoretical analysis of rate of release of solid drugs dispersed in solid matrices. J. Pharm. Sci. 1963, 52, 1145-1148.

17. Gohel, M. C.; Panchal, M. K.; Jogani, V. V. Novel Mathematical Method for Quantitative Expression of
Deviation from the Higuchi Model. AAPS PharmSciTech 2000, 1 (4), 20-25.

18. Brazel, C. S.; Peppas, N. A. Modeling of drug release from swellable polymers. Eur. J. Pharm. Biopharm. 2000, 49, 47-58.

19. Lapidus, H.; Lordi, N. G. Some factors affecting the release of a water-soluble drug from a compressed hydrophilic matrix. J. Pharm. Sci. 1966, 55, 840-843.

20. Korsmeyer, R. W.; Gurny, R.; Doelker, E.; Buri, P.; Peppas, N. A. Mechanisms of solute release from porous hydrophilic polymers. Int. J. Pharm. 1983, 15 (1), 25-35.

21. Hixson, A.W.; Crowel, J. H. Dependence of reaction velocity upon surface and agitation: theoretical considerations. Ind. Eng. Chem. 1931, 23, 923-931. 